\title{
Correction to: Dynamic Host Immune and Transcriptomic Responses to Respiratory Syncytial Virus Infection in a Vaccination-Challenge Mouse Model
}

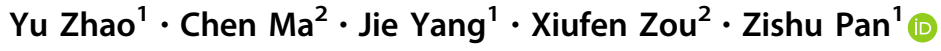

Published online: 13 July 2021

(C) Wuhan Institute of Virology, CAS 2021

\section{Correction to: Virologica Sinica}

https://doi.org/10.1007/s12250-021-00418-3

The original version of this article, published online on June 17, 2021, contained a mistake in Supplementary Table S5. The correct Supplementary Table S5 is given below.

The original article can be found online at https://doi.org/10.1007/ s12250-021-00418-3.

\section{Zishu Pan}

zspan@whu.edu.cn

1 State Key Laboratory of Virology, College of Life Sciences,

Wuhan University, Wuhan 430072, China

2 School of Mathematics and Statistics, Wuhan University, Wuhan 430072, China 